\title{
Microfabricated Implantable Parylene-Based Wireless Passive Intraocular Pressure Sensors
}

\author{
Po-Jui Chen, Student Member, IEEE, Student Member, ASME, Damien C. Rodger, Member, IEEE, \\ Saloomeh Saati, Mark S. Humayun, Member, IEEE, and Yu-Chong Tai, Fellow, IEEE
}

\begin{abstract}
This paper presents an implantable parylene-based wireless pressure sensor for biomedical pressure sensing applications specifically designed for continuous intraocular pressure (IOP) monitoring in glaucoma patients. It has an electrical $\mathrm{LC}$ tank resonant circuit formed by an integrated capacitor and an inductor coil to facilitate passive wireless sensing using an external interrogating coil connected to a readout unit. Two surface-micromachined sensor designs incorporating variable capacitor and variable capacitor/inductor resonant circuits have been implemented to realize the pressure-sensitive components. The sensor is monolithically microfabricated by exploiting parylene as a biocompatible structural material in a suitable form factor for minimally invasive intraocular implantation. Pressure responses of the microsensor have been characterized to demonstrate its high pressure sensitivity $(>7000 \mathrm{ppm} / \mathrm{mmHg})$ in both sensor designs, which confirms the feasibility of pressure sensing with smaller than $1 \mathrm{mmHg}$ of resolution for practical biomedical applications. A six-month animal study verifies the in vivo bioefficacy and biostability of the implant in the intraocular environment with no surgical or postoperative complications. Preliminary ex vivo experimental results verify the IOP sensing feasibility of such device. This sensor will ultimately be implanted at the pars plana or on the iris of the eye to fulfill continuous, convenient, direct, and faithful IOP monitoring.

[2008-0111]
\end{abstract}

Index Terms-Implantable microdevice, intraocular pressure (IOP), parylene, pressure sensor, wireless sensing.

\section{INTRODUCTION}

$\mathbf{G}$ LAUCOMA is a debilitating eye disease that chronically damages the optic nerve and results in loss of vision for tens of millions of people worldwide [1]. The disease is associated with abnormally accumulated intraocular fluid and

Manuscript received May 15, 2008; revised July 15, 2008. First published October 28, 2008; current version published December 4, 2008. This work was supported in part by the Engineering Research Centers Program of the National Science Foundation under Award EEC-0310723 and in part by Bausch and Lomb. An earlier version of this paper was presented at the 21st IEEE International Conference on Micro Electro Mechanical Systems, Tucson, AZ, January 13-17, 2008. Subject Editor S. Shoji.

P.-J. Chen is with the Department of Electrical Engineering, Division of Engineering and Applied Science, California Institute of Technology, Pasadena, CA 91125 USA (e-mail: pjchen@mems.caltech.edu).

D. C. Rodger is with the Keck School of Medicine, University of Southern California, Los Angeles, CA 90033 USA.

S. Saati and M. S. Humayun are with the Doheny Eye Institute, Los Angeles, CA 90033 USA, and also with the Keck School of Medicine, University of Southern California, Los Angeles, CA 90033 USA

Y.-C. Tai is with the Department of Electrical Engineering and the Department of Bioengineering, Division of Engineering and Applied Science, California Institute of Technology, Pasadena, CA 91125 USA

Color versions of one or more of the figures in this paper are available online at http://ieeexplore.ieee.org.

Digital Object Identifier 10.1109/JMEMS.2008.2004945 the resulting elevated intraocular pressure (IOP). Accordingly, successful IOP monitoring is crucial in the management of glaucoma patients as it is known as one of the most effective methods to evaluate the progression of this eye disease. Current clinical diagnosis involves contact or noncontact applanation tonometry for IOP recording. However, both modalities have difficulties in providing reliable and repeatable measurements and, particularly, in deployment for regular (e.g., daily) tracking, which impedes prompt detection and appropriate treatment for IOP spikes from its diurnal fluctuations considered as a separate risk factor to optic nerve damage [2]-[4]. Continuous IOP monitoring in glaucoma patients with high accuracy and high reliability is therefore a consistent need for ophthalmologists.

Telemetric sensing is one of the viable methods to accomplish continuous and faithful noncontact IOP measurements [5], [6]. It utilizes a transensor implant that registers environmental pressure variations inside the eye, so that the IOP can be directly measured by using an external reader wirelessly interrogating the implant. This methodology enables straightforward IOP sensing without involving further calculations which are derived from ocular mechanics as are used in applanation tonometry and which have large variation due to different dimensions and mechanical properties of individual eyes [7], [8]. In contrast to active sensing in which power transfer, size, and cost of the device are critical concerns [9], [10], passive sensing approaches have relatively flexible design considerations on the device side [11]. The first paradigm using this sensing method was reported in 1967 [12] where a capsulated sensor having an electrical $L C$ tank resonant circuit was implanted to the anterior chamber of the eye for IOP monitoring. With recent progress in microelectromechanical systems (MEMS) technology, miniaturized pressure sensors [13]-[17] have been developed using the $L C$ resonant circuit concept and the enabling microfabrication technologies for various wireless sensing applications, including transcutaneous pressure monitoring, intracranial pressure monitoring, and pressure monitoring of abdominal aortic aneurysms in addition to the proposed IOP monitoring. These devices serve as excellent examples showing the potential of using such wireless passive pressure transensors for the continuous measurement of physiological parameters in biomedical systems and human health care. The theory and model of wireless $L C$ sensing have been well established given the development of these reported pressure microsensors. On the technology side, however, all these MEMS devices to date involve delicate multiple wafer fabrication procedures with wafer bonding as the essential process to create the pressure-sensitive 


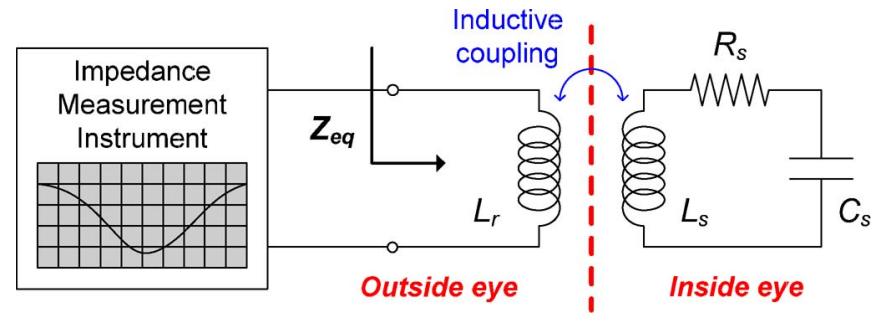

Fig. 1. Passive wireless IOP sensing concept. The implanted sensor can faithfully register pressure variations using corresponding electrical characteristic changes, which are measured using an external reader through a wireless inductive coupling link.

chamber of the variable capacitor, which creates challenges in adapting them to intraocular implants due to the small available space in the anterior chamber of the eye. Consequently, with the knowledge of exploiting parylene in integrated surfacemicromachined capacitive sensors [18], we have developed a monolithically microfabricated parylene-based wireless passive pressure sensor [19] in a form factor suitable for minimally invasive device implantation and placement at the pars plana or on the iris of the eye. Along with engineering specifications, design considerations are also focused on compatibility with surgical procedures to minimize complexity and time of operation for ease of device implantation in practice. Featuring parylene (polyparaxylene) as the biocompatible polymeric material, the pressure sensor is designed to be completely implantable in the intraocular environment so that long-term continuous IOP monitoring of glaucoma patients can be fulfilled.

\section{DESIGN}

\section{A. System Overview}

The conceptual schematic of the wireless IOP sensing system is shown in Fig. 1. The sensor implant is designed to have an electrical $L C$ tank resonant circuit with a corresponding resonant frequency represented as

$$
f_{s}=\frac{1}{2 \pi} \sqrt{\frac{1}{L_{s} C_{s}}-\frac{R_{s}^{2}}{L_{s}^{2}}} \cong \frac{1}{2 \pi \sqrt{L_{s} C_{s}}}, \quad \text { if } R_{s}^{2} \ll \frac{L_{s}}{C_{s}}
$$

where $L_{s}, C_{s}$, and $R_{s}$ are the inductance, capacitance, and resistance of the sensor, respectively. Using an external coil to build an inductive coupling link with the implanted sensor, the equivalent impedance viewed from the measurement instrument can be derived using circuit analysis as [17], [20]

$$
Z_{\text {eq }}=\frac{V}{I}=j 2 \pi f L_{r}\left[1+k^{2} \frac{\left(\frac{f}{f_{s}}\right)^{2}}{1-\left(\frac{f}{f_{s}}\right)^{2}+\frac{1}{Q_{s}} j \frac{f}{f_{s}}}\right]
$$

where $V$ and $I$ are the exciting voltage and current across the reader coil, respectively, $f$ is the excitation frequency, $Q_{s}=$ $R_{s}^{-1}\left(L_{s} C_{s}^{-1}\right)^{1 / 2}$ is the quality factor of the sensor at resonance, and $k$ is the coupling coefficient of the inductive link totally depended on physical geometries such as the planar size of the sensor and reader coils and the separation distance between

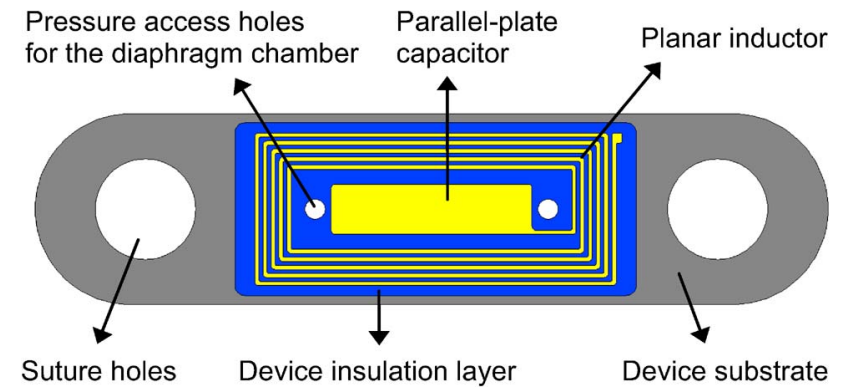

Fig. 2. Pressure sensor design (top view). The flexible diaphragm on top of the device area as the movable structure is not shown in this planar illustration.

the coils [15], [21]. Therefore, from (2), a phase-dip technique can be applied to wirelessly detect the resonant frequency of the sensor as the phase of the complex impedance $Z_{\text {eq }}$ drops to the minimum in the frequency scan from the measurement instrument, such as impedance analyzer, network analyzer, or others using customized electronics. When the sensor is excited at resonance, $Z_{\mathrm{eq}}$ becomes

$$
Z_{\text {eq }}=j 2 \pi f_{s} L_{r}\left(1+j k^{2} Q_{s}\right)
$$

and its phase-dip magnitude can be approximated as

$$
\Delta \phi \cong \tan ^{-1}\left(k^{2} Q_{s}\right)
$$

As long as the impedance phase dip is detectable in the frequency scan, the resonant frequency of the sensor can be accurately characterized. As a result, if the sensor implant has pressure-sensitive electrical components, its resonant frequency will be shifted based on external pressure variation, thus registering the in situ environmental pressure. This change can be interrogated using the external reader coil so that continuous wireless IOP monitoring can be accomplished.

\section{B. Device Structure}

The pressure sensor is designed as shown in Fig. 2 to facilitate the passive wireless pressure sensing scheme. It comprises a flexible diaphragm chamber integrated with parallel metal plates as an integrated capacitor and surrounding metal wires as a planar inductor to create the resonant circuit that communicates with the external reader. A dual-layer metal structure is employed to increase the overall inductance of the coil in the given device area. In the surgical design aspect, the device has a rectangular in-plane contour to minimize the required incision size during implantation along with smooth edges to prevent tissue irritation in the intraocular environment. Suture holes are located at both ends of the device for convenience in implant anchoring. In the engineering design aspect, the electrical characteristics of the sensor can be determined by using the following equations [14], [21], [22]. The inductance of the dual-layer planar inductor in rectangular conformation can be calculated as

$$
L_{s} \cong L_{s, \text { top }}+L_{s, \text { bot }}+2 M
$$


where $L_{s, \text { top }}$ and $L_{s, \text { bot }}$ are inductances from top and bottom metal layers, respectively, as

$$
\begin{aligned}
L_{s, \text { top }}= & L_{s, \text { bot }} \\
= & 0.02339 n^{2}\left[\left(s_{1}+s_{2}\right) \log \left(\frac{2 s_{1} s_{2}}{n D}\right)\right. \\
& \left.\quad-s_{1} \log \left(s_{1}+g\right)-s_{2} \log \left(s_{2}+g\right)\right] \\
& +0.01016 n^{2}\left[2 g-\frac{\left(s_{1}+s_{2}\right)}{2}+0.447 n D\right] \\
& -0.01016 n\left(s_{1}+s_{2}\right)(A+B) \quad \text { (in microhenrys) }
\end{aligned}
$$

where $n$ is the number of turns of the inductor, $s_{1}$ and $s_{2}$ are the average lengths of the two sides of the inductor (in inches), $g=\sqrt{s_{1}^{2}+s_{2}^{2}}$ (in inches), $D$ is the distance of the planar metal line separation plus metal line width (in inches), and $A$ and $B$ are constant coefficients determined by the winding geometry and the number of turns of the coil. The mutual inductance $M$ is generated by intrinsic magnetic coupling between the top and bottom metal wires of the inductor and can be calculated as

$M=0.00254 l\left[2.303 \log \left(\frac{l}{g}\right)-1+\frac{2 g}{l}\right], \quad$ if $l \gg g$

(in microhenrys)

where $l$ is the total length of the dual-layer metal wires $(l / 2$ for each layer) (in inches) and $g$ is the separation distance between the top and bottom metal layers of the inductor (in inches). The integrated inductor imperatively has a series resistance dominating $R_{s}$, and its value with consideration of the highfrequency skin effect can be calculated as

$$
R_{s}=\frac{\rho l}{w \delta\left(1-e^{-h / \delta}\right)}
$$

where $\rho$ is the electrical resistivity of the metal, $w$ and $h$ are the metal line width and height, respectively, and $\delta$ is the frequency-dependent metal skin depth given by

$$
\delta=\sqrt{\frac{\rho}{\pi f \mu}}
$$

where $\mu$ is the magnetic permeability of the metal. Finally, the capacitance of the sensor can be expressed as

$$
C_{s}=C_{s, g}+C_{s, p}
$$

where $C_{s, g}$ is the capacitance determined by the integrated parallel metal plates with a gap separation at the center of the sensor and $C_{s, p}$ is the parasitic/stray capacitance generated by the other components in the entire device (e.g., such from the metal-dielectric-metal structure realizing the dual-layer inductor wires). It is clear from (5)-(7), and (10) that the electrical characteristics of the sensor can vary with respect to geometrical changes in the movable structures. Therefore, a deformable free-standing diaphragm embedding electrical components is employed to realize the transensor device. The
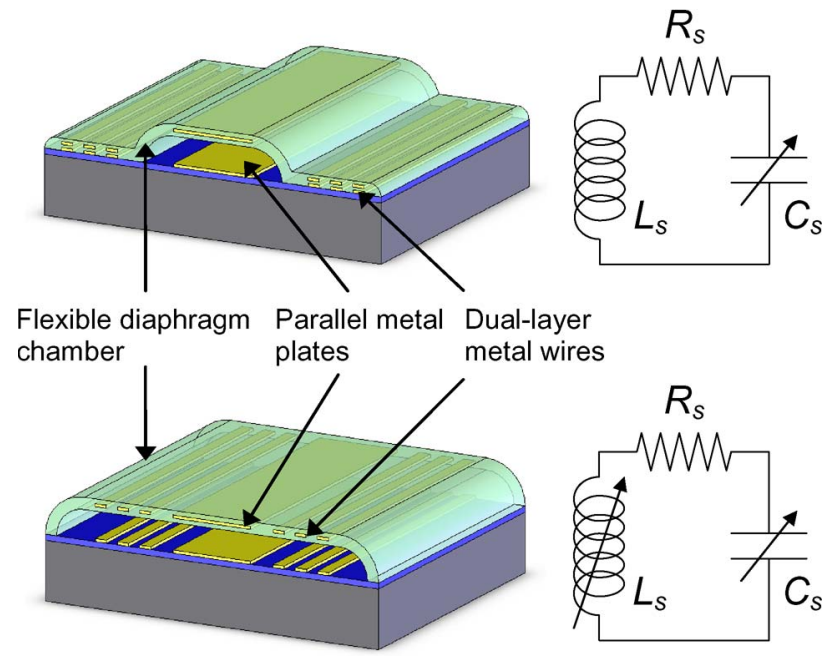

Fig. 3. Sensor design variations (cross-sectional view; not to scale). (Top) Variable capacitor. (Bottom) Variable capacitor/inductor. The deformable diaphragm chamber with respect to pressure changes realizes pressure-sensitive electrical characteristics of the sensors.

deformation profile of a rectangular diaphragm with four fixed edges under a pressure loading can be estimated as in [23], where in the small-deflection case, the maximum diaphragm deformation at the center can be calculated by [24]

$$
y_{\max }=\left[\frac{C_{1} a^{4}}{D}\right] \Delta P
$$

where $\Delta P$ is the pressure difference across the diaphragm, $a$ is half the length of the short edge of the rectangular diaphragm, $D=E t^{3} /\left[12\left(1-\nu^{2}\right)\right]$ is the flexural rigidity of the diaphragm in which $E$ is the Young's modulus and $\nu$ is the Poisson's ratio of the material, $t$ is the diaphragm thickness, and $C_{1}$ is a constant coefficient determined by the geometry and boundary conditions of the diaphragm. In the large-deflection case, where the assumption $(y \ll t)$ is not valid, $y_{\max }$ can be calculated using the load-deflection relationship as [25], [26]

$$
\left(\frac{C_{2} \sigma t}{a^{2}}\right) y_{\max }+\left(\frac{C_{3} E t}{a^{4}}\right) y_{\max }^{3}=\Delta P
$$

where $\sigma$ is the internal stress of the diaphragm and $C_{2}$ and $C_{3}$ are constant coefficients determined by the geometry and material property (Poisson's ratio) of the diaphragm. Incorporating such electrical-mechanical-coupled effect, a pressure-sensitive variable capacitor can be realized as shown in Fig. 3 by arranging the bottom plate on the substrate and the top plate in the diaphragm. Additionally, instead of having a fixed inductor surrounding the variable capacitor, a variable inductor can be realized by embedding the top metal wire layer in the diaphragm to alter the intrinsic mutual inductance of the dual-layer inductor for further enhanced overall pressure sensitivity. These two designs (variable capacitor and variable capacitor/inductor) are implemented on a monolithic substrate with the use of surfacemicromachining technology so as to have less fabrication complexity compared with wafer bonding technology. The pressure reference can be encapsulated inside the diaphragm chamber after sealing the backside of the device at a constant pressure 
(a)
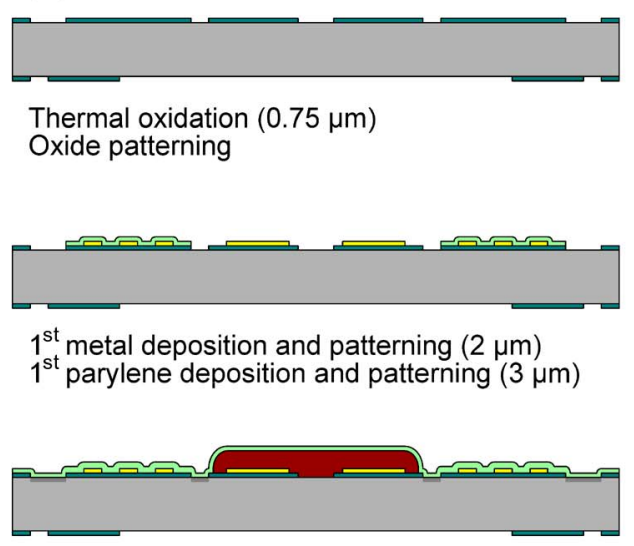

Sacrificial PR coating and patterning $(6 \mu \mathrm{m})$ $\mathrm{XeF}_{2}$ silicon roughening $2^{\text {nd }}$ parylene deposition and patterning $(2 \mu \mathrm{m})$
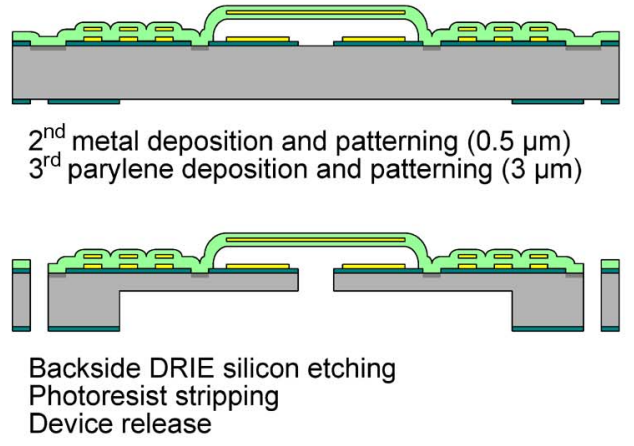

(b)
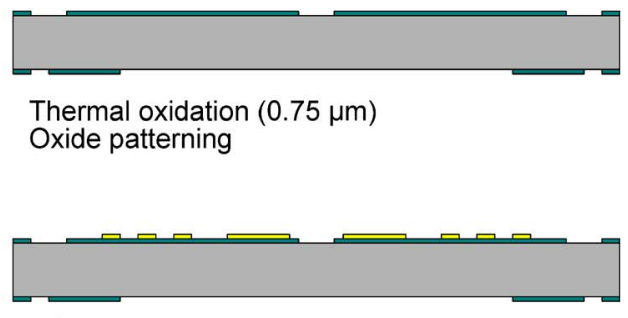

$1^{\text {st }}$ metal deposition and patterning $(2 \mu \mathrm{m})$

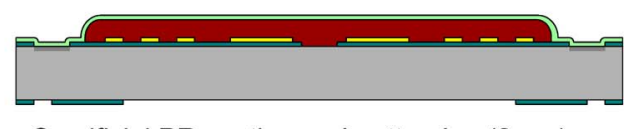

Sacrificial PR coating and patterning $(6 \mu \mathrm{m})$ $\mathrm{XeF}_{2}$ silicon roughening

$1^{\text {st }}$ parylene deposition and patterning $(2 \mu \mathrm{m})$
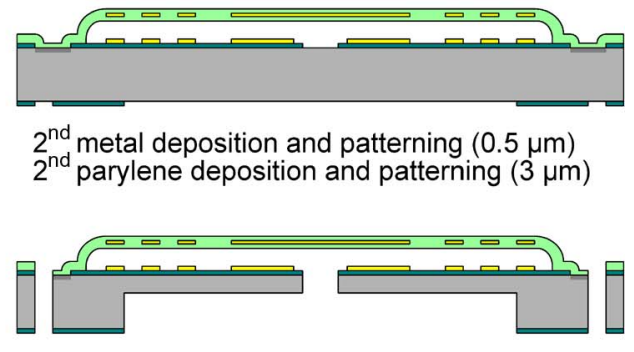

Backside DRIE silicon etching

Photoresist stripping

Device release

\section{Silicon $\square \mathrm{SiO}_{2} \square \mathrm{Ti} / \mathrm{Au} \square$ Photoresist $\square$ Parylene}

Fig. 4. Fabrication process flow of microsensors. (a) Variable capacitor design. (b) Variable capacitor/inductor design.

for gauge pressure sensing. All device structures including oxidized and parylene-coated silicon substrate, titanium/gold metal lines and plates, and flexible parylene diaphragm have sufficient biocompatibilty to ensure the feasibility and reliability of the long-term operation of the device implant in the intraocular environment. Parylene C (poly-chloro-p-xylylene) is selected as the diaphragm material because of its mechanical flexibility (Young's modulus $\sim 4 \mathrm{GPa}$ ), CMOS/MEMS process compatibility, and biocompatibility (Food and Drug Administration approved United States Pharmacopeia Class VI grade) [27], [28]. Its room-temperature conformal deposition nature allows large surface-micromachined diaphragm chambers to be formed on a monolithic substrate. The dielectric behavior of parylene (dielectric constant $\sim 2.95$ at $1 \mathrm{MHz}$ ) also favors its use as an insulator between metal layers. Given the aforementioned models and materials, the overall sensing performance can be estimated to meet the required specification of miniature pressure variation measurement $( \pm 1 \mathrm{mmHg})$ for accurate IOP monitoring.

\section{FABRICATION}

The fabrication process of the wireless pressure sensor is shown in Fig. 4. It started with thermally growing and patterning a $0.75-\mu \mathrm{m}$-thick oxide on a double-side-polished sil- icon wafer using buffered hydrofluoric acid (BHF, Transene Company Inc., Danvers, MA) and photoresist as a mask. AZ series photoresist (Clariant Corporation, Charlotte, NC) was used in this step as well as in the following steps throughout the described microfabrication processes. The frontside oxide serves as an insulation layer between the silicon substrate and the surface-micromachined structures, and the backside oxide defines the recess and release boundaries of the device. A thick titanium/gold $(200 \AA / 2 \mu \mathrm{m})$ layer was deposited using e-beam evaporation and patterned using standard metal etching techniques to form the bottom half of the electrical components of the sensor. Thick metals are preferred in order to reduce the overall electrical resistance of the metal lines. Although metals with higher thickness could be deposited using electroplating, evaporated metals were used in this work because of their superior material quality for biomedical implant applications and fabrication compatibility with subsequent surfacemicromachining processes. A parylene layer was then coated on the bottom metal of the sensor in the variable capacitor design for insulation purposes between the dual-layer metal wires, while an air gap was used for the sensor in the variable capacitor/inductor design. Parylene deposition was conducted in a Cookson Electronics PDS 2010 system (Specialty Coating Systems Inc., Indianapolis, IN). A via for metal contact was created in the parylene layer after patterning it using oxygen 


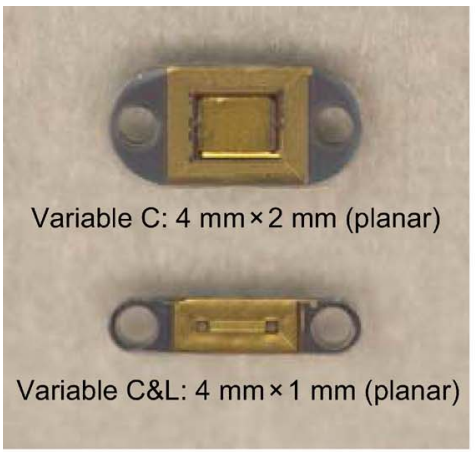

(a)

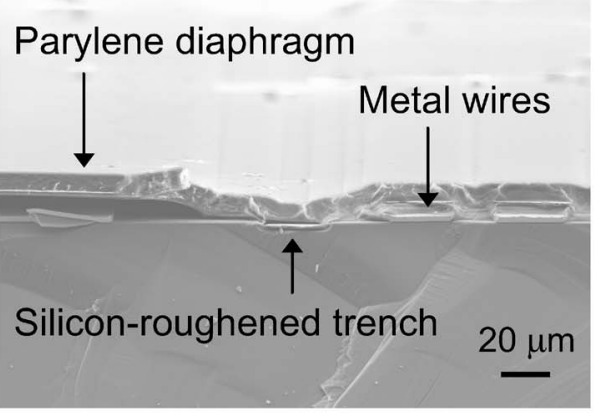

(b)

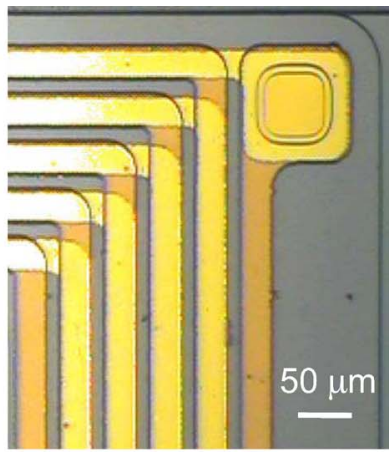

(c)

Fig. 5. Images of microfabricated devices. (a) Full-scale photograph of the sensors (top view). (b) SEM image of the sensor (cross-sectional view). (c) Micrograph of the sandwiched metal-parylene-metal structure showing the inductor wiring and the via (top view). (b) and (c) are images of the sensor in variable capacitor design as example.

plasma and photoresist as the mask. A sacrificial layer of photoresist was then coated and patterned on the wafers so as to define the enclosed space of the pressure-sensitive diaphragm chamber. Gas-phase xenon difluoride $\left(\mathrm{XeF}_{2}\right.$, Nesca Corporation, Pretoria, South Africa) silicon roughening was preformed for physically strengthened adhesion between the final surface-micromachined structures and the substrate [29]. Afterwards, sandwiched parylene-metal-parylene layers were deposited and patterned including another thick e-beamevaporated titanium/gold $(200 \AA / 0.5 \mu \mathrm{m})$ layer to realize the flexible diaphragm chambers with variable capacitor or capacitor/inductor structures by having different patterns of the sacrificial photoresist layer in different sensor designs. Finally, the devices were released after performing a silicon deep reactive-ion etching (DRIE) in a PlasmaTherm SLR system (Unaxis Inc., St. Petersburg, FL) on the backside of the wafer followed by photoresist stripping with acetone. Backside recesses and through holes as necessary features were also created after DRIE. Supercritical carbon dioxide $\left(\mathrm{CO}_{2}\right)$ drying in an Autosamdri-815 system (Tousimis Research Corporation, Rockville, MD) was used to eliminate the stiction of the freestanding parylene-metal-parylene diaphragm. Fig. 5 shows the microfabricated parylene-based pressure sensors in both designs, in a form factor smaller than $4 \mathrm{~mm} \times 2 \mathrm{~mm} \times 1 \mathrm{~mm}$ so suitable for intraocular implantation.

In order to achieve both successful device release and packaging, a special two-step etch mask consisting of oxide and photoresist was used for the backside DRIE. By controlling the heights of this two-step mask and the associated etch time, a backside recess was formed on the substrate along with through-wafer etches at locations of the photoresist release holes beneath the parylene diaphragm chamber, end suture holes, and release boundaries of the device. The backside recess enables gauge pressure sensing of the devices. For gas-sealed MEMS gauge pressure sensors, the change of encapsulated pressure reference due to diaphragm deflection and the corresponding change of volume should be considered [30] as shown in Fig. 6. Derived from the ideal gas law, the increase of the pressure reference can be written as [16]

$$
\Delta P_{0}=\frac{n R T}{V_{0}-\Delta V}-P_{0}
$$

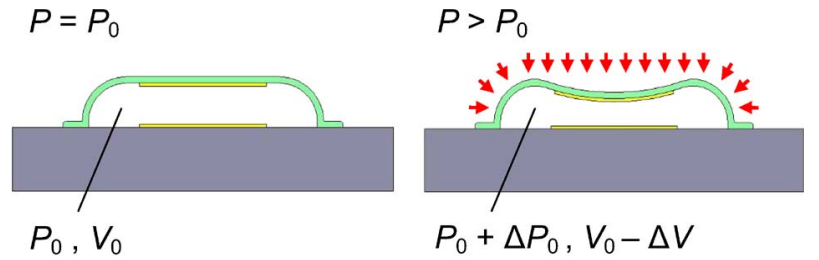

(a)

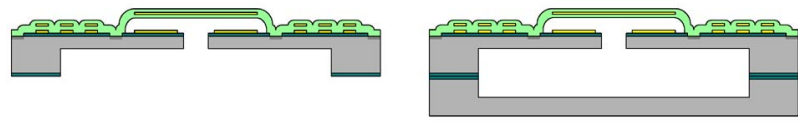

(b)

Fig. 6. Volume-change effect in gas-sealed MEMS gauge pressure sensors. (a) The encapsulated gas volume and pressure are variable due to diaphragm deformation. (b) Method to reduce such effect by attaching the sensor backto-back to a nonelectric piece to increase the reference volume $V_{0}$ (variable capacitor design as example).

where $n$ is the number of moles of gas particles, $R$ is the universal gas constant, $T$ is the environmental temperature, $\Delta V$ is the magnitude of change of volume, and $V_{0}$ and $P_{0}$ are the originally encapsulated volume and pressure of the sensor, respectively. As a result, the change of reference pressure would influence the diaphragm deflection, and the resultant pressure sensing behavior thus needs to be reduced if it is comparable with the pressure difference to be measured. For the microsensors fabricated in this work, a large gas volume can be encapsulated inside the device after sealing the $250-\mu \mathrm{m}$-deep backside recess in addition to that from the surface-micromachined diaphragm chamber, which effectively increases the resultant pressure sensing capacity after packaging. For demonstration purposes, the fabricated sensor device in this work was attached back-to-back to a nonelectronic parylene-coated silicon piece with identical geometrical dimensions under atmospheric pressure $(1 \mathrm{~atm})$ at room temperature. The influence on pressure sensing from such volume-change effect was then controlled to less than $100 \mathrm{ppm} / \mathrm{mmHg}$, insignificant compared with the results from designed compliant diaphragm deflections. The overall device thickness can definitely be trimmed by thinning down the silicon pieces during fabrication to result in smaller device sizes if required. After packaging, a thin conformal parylene layer could be selectively coated on the entire device to enhance its biocompatibility for medical use. 
TABLE I

Electrical Parameters of the MicrofabricAted WIRELESS PRESSURE SENSORS

\begin{tabular}{ccc}
\hline & Variable C & Variable C\&L \\
\hline Inductance & $0.78 \mu \mathrm{H}$ & $0.36 \mu \mathrm{H}$ \\
Capacitance & $8.5 \mathrm{pF}$ & $3.1 \mathrm{pF}$ \\
Resistance & $112 \Omega$ & $72 \Omega$ \\
Resonant frequency & $\sim 62 \mathrm{MHz}$ & $\sim 150 \mathrm{MHz}$ \\
Quality factor & $\sim 3$ & $\sim 5$ \\
\hline
\end{tabular}

\section{RESULTS AND DiscuSSION}

The microfabricated devices were tested both on-bench and in vivo to characterize their electrical, physical, and surgical/ biological behaviors. During testing, an HP 4195A network/ spectrum analyzer (HP/Agilent Technologies Inc., Santa Clara, CA) under standard settings was utilized in connection with a 1.5-mm-diameter hand-wound coil made out of standard insulated copper wire to serve as the external reader for wireless sensing demonstration. Measured equivalent impedance spectra data were stored in a personal computer using the LabVIEW program (National Instruments Corporation, Austin, TX) for pressure measurement analysis. Testing results are described in the following sections.

\section{A. Electrical Characterization}

Electrical parameters of the sensors were first obtained by analyzing the measurement data from both the actual devices with the external readout method and several testing structures through on-chip probing. Table I lists the experimental results in good agreement with theoretical calculations. The resistance, inductance, and capacitance of the sensor in the variable capacitor design were all higher than those of the sensor in the variable capacitor/inductor design due to larger device area. The duallayer metal wires in proximity across the parylene dielectric in the variable capacitor design gave a substantial stray capacitance to the sensor. Although the low quality factor $\left(Q_{s}<10\right)$ and small integrated coil size of the sensors fundamentally limit the sensing distance of the wireless system, the sensor signal was still detectable as shown in Fig. 7, which validates the feasibility of the impedance phase-dip measurement technique as the sensing scheme. Being different from the ideal case, the phase of the impedance $Z_{\text {eq }}$ dipped to the minimum at the frequency $f_{\min }$, which is highly correlated to $f_{s}$ and can be expressed as [20]

$$
f_{\min }=f_{s}\left(1+\frac{k^{2}}{4}+\frac{1}{8 Q^{2}}\right)
$$

while the discrepancy was less than $1 \%$ given the negligible term from the small coil coupling coefficient effect compared with the term from the quality factor effect in this work. The maximum sensing distance where the phase dip $\Delta \phi<0.1^{\circ}$, determined by the noise floor from the testing system, was confirmed to be approximately $2 \mathrm{~mm}$ for both sensor designs.

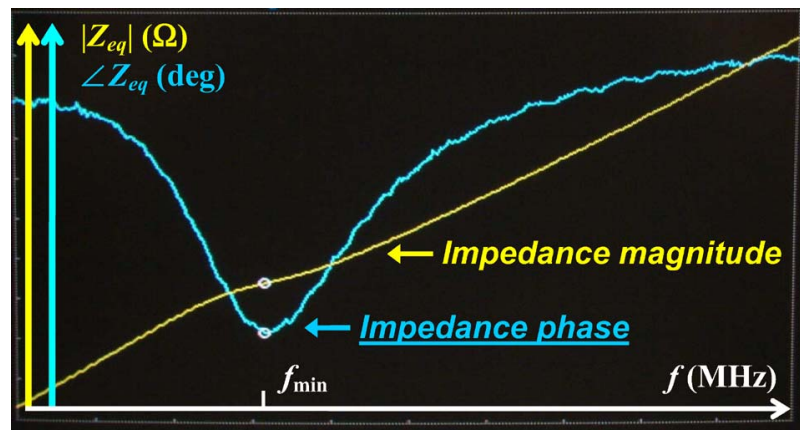

Fig. 7. Representative captured frequency scan of the equivalent impedance $Z_{\text {eq }}$ of the reader coil coupled with the microsensor.

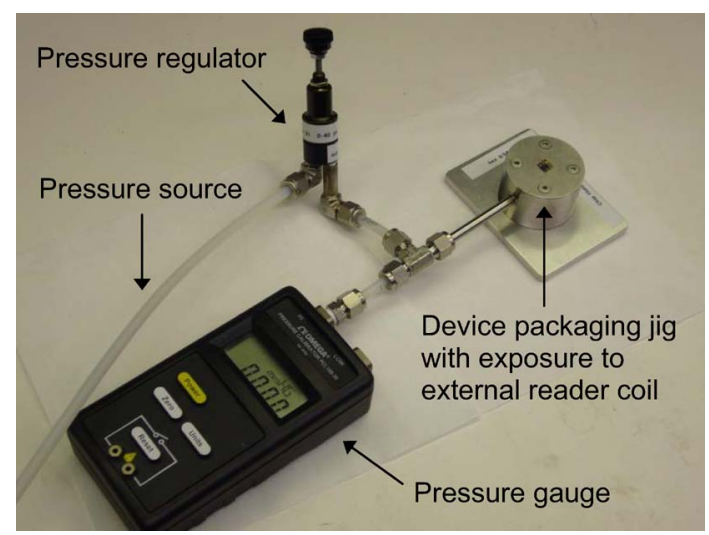

Fig. 8. Pressure testing setup for on-bench device characterization.

Other than reader optimization [31]-[33], the following strategies directly related to sensor development can be implemented to improve the sensing distance and should be included in future work: 1) increase the integrated coil size/planar geometry $(k \uparrow) ; 2)$ increase the metal wire thickness $\left(R_{s} \downarrow\right)$; 3) increase the insulating oxide thickness and/or modify the substrate material $\left(C_{s} \downarrow\right)$; and 4) include ground shield patterns [34] which can be potentially utilized as inductor interlayer shield patterns $\left(C_{s} \downarrow\right)$.

\section{B. On-Bench Pressure Test}

After electrical characterization, the sensors were tested on chip to measure their pressure responses. Pressure differences were generated by supplying pressurized air to the inside of the sensor diaphragm chamber through a customized packaging jig connected to a controllable pressure regulation system as shown in Fig. 8 with constant environmental pressure outside the diaphragm chamber. A commercial off-chip pressure regulator and a pressure gauge were used to control the pressurization with $0.5-\mathrm{mmHg}$ tuning resolution. The external reader coil was aligned above the packaged device on the same axis using a manipulation stage for in situ wireless sensing. This testing configuration was applied to condition the sensors for testing convenience without losing the fidelity of the sensor performance because the volume-change effect can be neglected given the fabricated device dimensions. Experimental results, as shown in Fig. 9, successfully verified the feasibility of wireless pressure sensing with the sensor in the variable capacitor design 


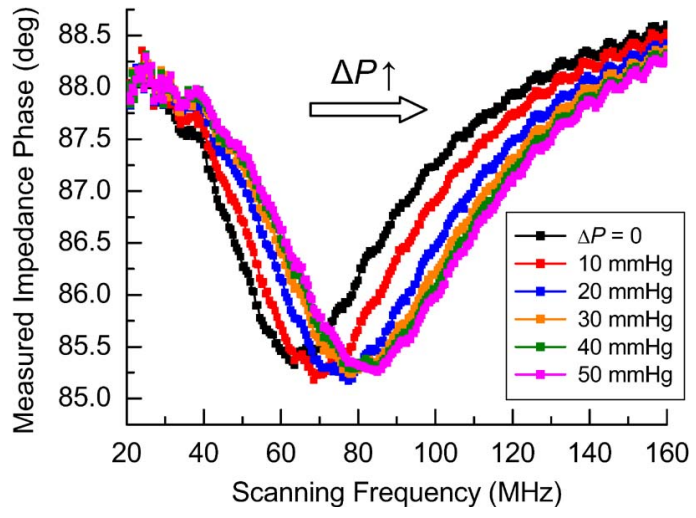

Fig. 9. Overlay plot of measured phase-dip curves of the sensor (variable capacitor design as example) in on-bench wireless pressure sensing. Here, $\Delta P=P_{\text {inside sensor }}-P_{\text {outside sensor }}$.

as an example. As the pressure inside the diaphragm chamber increases, the diaphragm is deformed outward and moves the embedded metal components away from the bottom substrate, leading to a decrease in the overall capacitance/inductance and an increase in the resonant frequency of the sensor. For the sensors in the variable capacitor design, the normalized shifted resonant frequency can be written as

$$
\begin{aligned}
\frac{f_{\min }(\Delta P)}{f_{\min }(\Delta P=0)} & =\frac{\frac{1}{2 \pi \sqrt{L_{s}\left(C_{s}-\Delta C_{s}\right)}}}{\frac{1}{2 \pi \sqrt{L_{s} C_{s}}}} \\
& =\sqrt{1+\frac{\Delta C_{s}}{C_{s}}}, \quad \text { if } \Delta C_{s} \ll C_{s}
\end{aligned}
$$

where $\Delta C_{s}$ is the changed capacitance due to diaphragm deflection. It can be further derived as

$$
\frac{f_{\min }(\Delta P)}{f_{\min }(\Delta P=0)}=(1+\alpha y)^{1 / 2}
$$

where $\alpha$ is a constant coefficient based on the material properties and physical dimensions of the device. Assuming a small diaphragm deflection which is proportional to pressure difference by (11), the relationship can be finally derived as

$$
\frac{f_{\min }(\Delta P)}{f_{\min }(\Delta P=0)}=\left(1+\alpha^{\prime} \Delta P\right)^{1 / 2}
$$

where $\alpha^{\prime}$ is a parameter incorporating the mechanical behavior of the diaphragm. For the sensors in the variable capacitor/ inductor design, the normalized shifted resonant frequency can be written as

$$
\begin{aligned}
\frac{f_{\min }(\Delta P)}{f_{\min }(\Delta P=0)} & =\frac{\frac{1}{2 \pi \sqrt{\left(L_{s}-\Delta L_{s}\right)\left(C_{s}-\Delta C_{s}\right)}}}{\frac{1}{2 \pi \sqrt{L_{s} C_{s}}}} \\
& =\sqrt{1+\frac{\Delta L_{s}}{C_{s}}} \sqrt{1+\frac{\Delta C_{s}}{C_{s}}}, \quad \text { if } \begin{array}{l}
\Delta L_{s} \ll L_{s} \\
\Delta C_{s} \ll C_{s}
\end{array}
\end{aligned}
$$

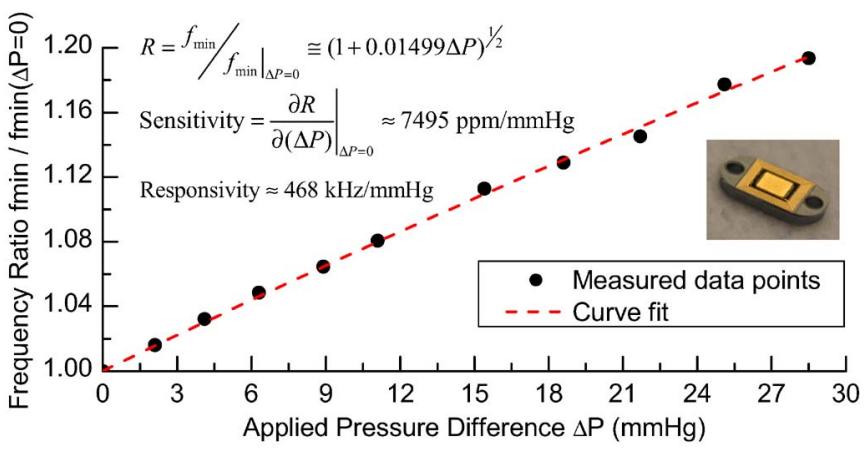

(a)

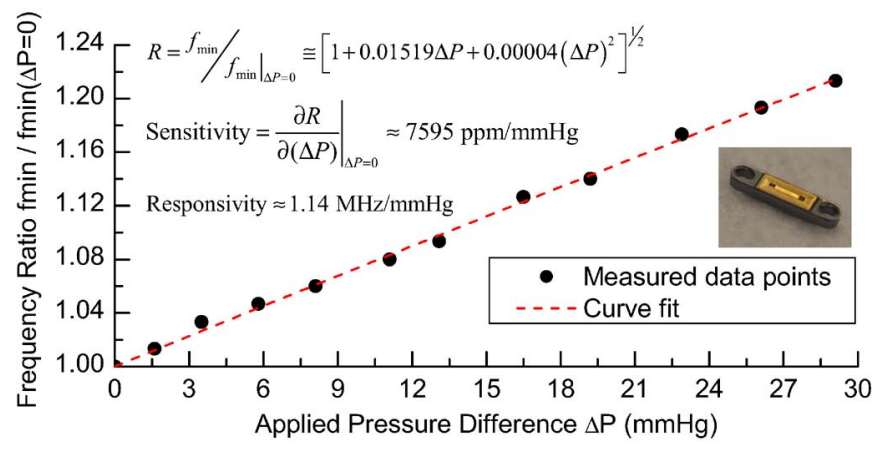

(b)

Fig. 10. On-bench pressure testing results of the sensors. (a) Sensor in variable capacitor design. (b) Sensor in variable capacitor/inductor design.

where $\Delta L_{s}$ is the changed inductance due to diaphragm deflection. Similar procedures can be used with the small deflection assumption to derive the dependence of the normalized shift resonant frequency on pressure difference as

$$
\frac{f_{\min }(\Delta P)}{f_{\min }(\Delta P=0)}=\left[1+\beta_{1} \Delta P+\beta_{2}(\Delta P)^{2}\right]^{1 / 2}
$$

where $\beta_{1}$ and $\beta_{2}$ are parameters incorporating coupled capacitance and inductance variations from the mechanical behavior of the diaphragm, implying a more sensitive pressure response from this design. The measurement results from the sensors in both designs as shown in Fig. 10 were well fit using (17) and (19) in 0-30-mmHg pressure operation range. Data analysis confirmed that the microfabricated sensors achieved excellent pressure sensing performance with high sensitivities $(>7000 \mathrm{ppm} / \mathrm{mmHg}$ ), providing readouts with resolutions smaller than $1 \mathrm{mmHg}$ so as to sufficiently cover all pressure variations of interest for practical IOP monitoring. Highly sensitive pressure responses were obtained based on the high compliance of the parylene flexible diaphragm and high resonant frequency of the sensors. In spite of its smaller parylene diaphragm, the sensor in the variable capacitor/inductor design has a comparable sensitivity result with that in the other design, showing the possibility of using devices in a smaller form factor while achieving the required sensing performance. Corrections would need to be made on (17) and (19) to accommodate large diaphragm deflections by (12) if the diaphragm experiences large pressure loadings, as this pressure-sensitivity-reduced effect can be observed in Fig. 9 where the phase-dip frequency shift was smaller when the applied pressure difference was 
TABLE II

MeASUREd CHARACTERISTICS OF THE MicrofabricATED WIRELESS PRESSURE SENSORS

\begin{tabular}{ccc}
\hline & Variable C & Variable C\&L \\
\hline Planar dimensions & $4 \mathrm{~mm} \times 2 \mathrm{~mm}$ & $4 \mathrm{~mm} \times 1 \mathrm{~mm}$ \\
Resonant frequency & $\sim 62 \mathrm{MHz}$ & $\sim 150 \mathrm{MHz}$ \\
Quality factor & $\sim 3$ & $\sim 5$ \\
Pressure sensitivity & $7495 \mathrm{ppm} / \mathrm{mmHg}$ & $7595 \mathrm{ppm} / \mathrm{mmHg}$ \\
Pressure responsivity & $0.47 \mathrm{MHz} / \mathrm{mmHg}$ & $1.14 \mathrm{MHz} / \mathrm{mmHg}$ \\
\hline
\end{tabular}

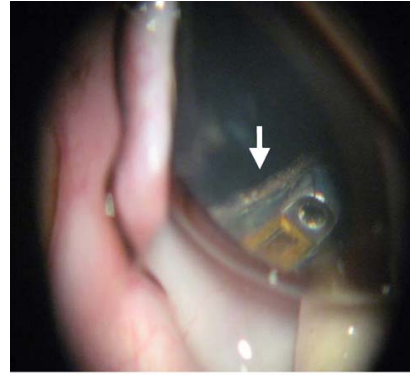

(a)

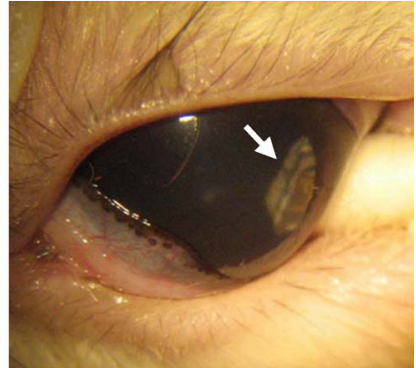

(b)
Fig. 11. In vivo device testing using rabbit model to verify long-term biocompatibility of the intraocular implant. (a) Three months after surgery. (b) Six months after surgery. Arrows indicate the implant as observed through the cornea.

closer to $50 \mathrm{mmHg}$. Table II summarizes several important characteristics of the microfabricated wireless pressure sensors presented in this paper.

\section{In Vivo Implantation Test}

In vivo device implantation testing was conducted using live rabbit eyes as the model to evaluate the bioefficacy and biostability of the sensor implant. The fully packaged microdevices with the bottom sealing piece were sterilized using ethylene oxide gas and implanted at the pars plana site in the eyes of two rabbits. All animal procedures in this study conformed to the Association for Research in Vision and Ophthalmology Statement on the Use of Animals in Ophthalmic and Vision Research. A small 2-mm angled razor blade incision was made to insert the implant into the eye. Clinical procedures utilizing the end suture holes of the devices were developed to facilitate fast and robust device anchoring during implantation. The operation could be completed within 15 min, curtailing the occurrence of surgical complications. Long-term follow-up observation with results as shown in Fig. 11 indicated that no postoperative complications including inflammatory response or tissue encapsulation/fibrosis were observed throughout the six-month study, which provides evidence of device biocompatibility in the intraocular environment. Extensive animal studies including demonstration of in vivo wireless pressure sensing and optimal intraocular device placement will be performed in the near future.

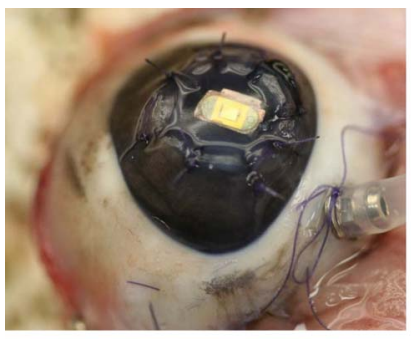

(a)

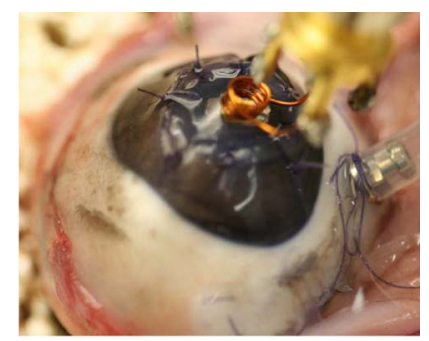

(b)
Fig. 12. Ex vivo device testing using porcine eye model to demonstrate the wireless pressure sensing behavior of the sensor implant. (a) Test model with the sensor inside the eye. (b) Wireless sensing with the external coil placed in front of the eye model.

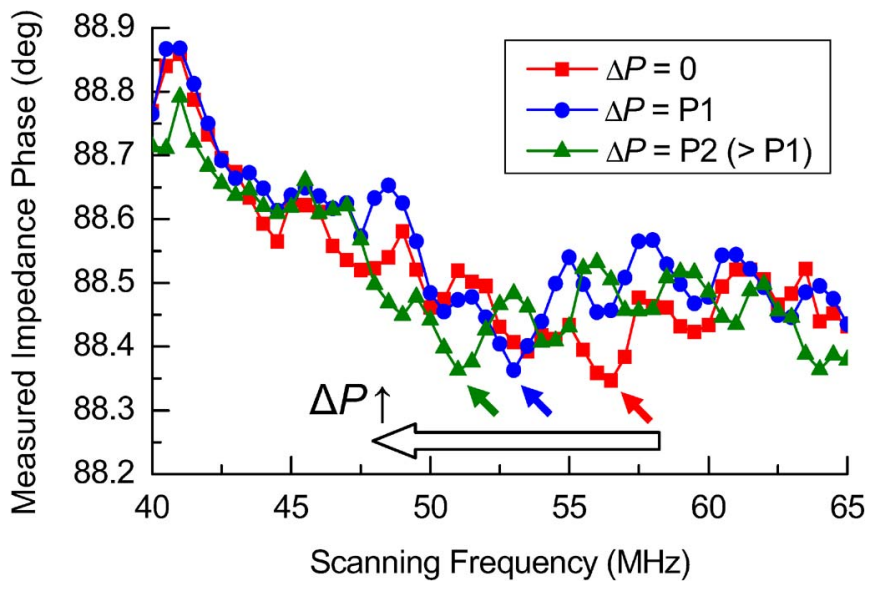

Fig. 13. Overlay plot of measured phase-dip curves of the sensor (variable capacitor design as example) in ex vivo wireless pressure sensing. The small arrows indicate the phase-dip shift with respect to the simulated IOP increase. Here, $\Delta P=P_{\text {outside sensor }}-P_{\text {inside sensor }}$.

\section{Ex Vivo Pressure Sensing Test}

The pressure sensor was implanted into an enucleated porcine eye as shown in Fig. 12 in order to conduct acute ex vivo pressure sensing characterization. The microfabricated sensor was placed under the cornea so as to have the best proximity to the external reader coil. Hence, a corneal flap incision was made with the use of sutures to restore the environment to an enclosed intraocular space after implantation. A catheter was then inserted to the eye model interfacing with an external infusion-based pressurization setup to simulate a situation of IOP variation. Although not ideal, the experimental results as shown in Fig. 13 demonstrated the feasibility of using this microsensor for wireless pressure sensing in such an isolated environment. The measured phase-dip frequency of the equivalent impedance dropped more than $15 \%$ as the sensor implant experienced a substantial environmental permittivity change in the liquid medium as compared with air [17]. The measurement noise in the plots resulted from the close proximity of the reader coil to the cornea necessary for interrogating the implant given the short sensing distance and could be greatly improved by having a longer sensing distance from stronger sensor-reader coil coupling. The results revealed another issue that the pressure sensitivity was difficult to be characterized in this testing setup because stable pressure control was not achieved due to 
protocols in place at the time of surgery. For more systematic ex vivo and in vivo pressure sensing studies, future work will also focus on the development of surgical procedures and tools having improved compatibility with such implant paradigm.

\section{CONClusion}

A novel microfabricated implantable wireless passive IOP sensor has been successfully developed, featuring parylene as the biocompatible/implantable structural material. Complete design, fabrication, and characterization of the microsensors are described. Sensors with electrical resonant circuitry in pressuresensitive variable capacitor and variable capacitor/inductor designs were implemented to facilitate wireless pressure sensing through an inductive coupling link with an external reader. A low-temperature multilayer polymer/metal micromachining technology enables integrated sensor components fabricated on a monolithic substrate without the need for a multiwafer bonding process. Sensors with implantable planar dimensions $(4 \mathrm{~mm} \times 2 \mathrm{~mm}$ in variable capacitor and $4 \mathrm{~mm} \times 1 \mathrm{~mm}$ in variable capacitor/inductor designs) as well as surgical features were microfabricated in a suitable form factor for intraocular implantation. Experimental measurement results were obtained and shown to be in good agreement with design parameters. The on-bench device testing with the sensors in both designs characterized the sensor performance with more than $7000 \mathrm{ppm} / \mathrm{mmHg}$ of sensitivity $(\sim 7495 \mathrm{ppm} / \mathrm{mmHg}$ in variable capacitor design and $\sim 7595 \mathrm{ppm} / \mathrm{mmHg}$ in variable capacitor/inductor design), resulting in successful pressure sensing with smaller than $1 \mathrm{mmhg}$ of resolution in a measurement range of more than $0-30 \mathrm{mmHg}$. The biocompatibility of the device implant was successfully verified in vivo through a sixmonth animal study, and the pressure sensing feasibility after implantation was preliminarily verified by ex vivo experiments. Given the demonstrated engineering and surgical/biological results, it is evident that the microsensors can be utilized for long-term continuous IOP monitoring in glaucoma patients. Future work will be focused on improving the sensing distance of these wireless pressure sensors for practical applications.

\section{ACKNOWLEDGMENT}

The authors would like to thank W. Li for her assistance on data acquisition of electrical impedance measurements, Dr. R. Agrawal and Dr. R. Varma for their valuable comments on surgical procedures, and T. Roper for his fabrication assistance.

\section{REFERENCES}

[1] M. Sherwood, "Glaucoma," in Ophthalmology, 2nd ed. M. Yanoff and J. S. Duker, Eds. St. Louis, MO: Mosby, 2004, pp. 1413-1473.

[2] S. Asrani, R. Zeimer, J. Wilensky, D. Gieser, S. Vitale, and K. Lindenmuth, "Large diurnal fluctuations in intraocular pressure are an independent risk factor in patients with glaucoma," J. Glaucoma, vol. 9, no. 2, pp. 134-142, Apr. 2000.

[3] E. Hughes, P. Spry, and J. Diamond, "24-hour monitoring of intraocular pressure in glaucoma management: A retrospective review," J. Glaucoma, vol. 12 , no. 3, pp. 232-236, Jun. 2003.

[4] R. Puers, G. Vandevoorde, and D. D. Bruyker, "Electrodeposited copper inductors for intraocular pressure telemetry," J. Micromech. Microeng., vol. 10 , no. 2 , pp. 124-129, Jun. 2000.
[5] J. W. McLaren, R. F. Brubaker, and J. S. FitzSimon, "Continuous measurement of intraocular pressure in rabbits by telemetry," Investig. Ophthalmol. Vis. Sci., vol. 37, no. 6, pp. 966-975, May 1996.

[6] K. C. Katuri, S. Asrani, and M. K. Ramasubramanian, "Intraocular pressure monitoring sensors," IEEE Sensors J., vol. 8, no. 1, pp. 12-19, Jan. 2008.

[7] C. R. Ethier, M. Johnson, and J. Ruberti, "Ocular biomechanics and biotransport," Annu. Rev. Biomed. Eng., vol. 6, pp. 249-273, Feb. 2004.

[8] I. G. Pallikaris, G. D. Kymionis, H. S. Ginis, G. A. Kounis, and M. K. Tsilimbaris, "Ocular rigidity in living human eyes," Investig. Ophthalmol. Vis. Sci., vol. 46, no. 2, pp. 409-414, Feb. 2005.

[9] W. Mokwa and U. Schnakenberg, "Micro-transponder systems for medical applications," IEEE Trans. Instrum. Meas., vol. 50, no. 6, pp. 1551-1555, Dec. 2001.

[10] W. Mokwa, "Medical implants based on microsystems," Meas. Sci. Technol., vol. 18, no. 5, pp. R47-R57, May 2007.

[11] S. T. J. Kaiser, "Passive telemetric readout system," IEEE Sensors J., vol. 6, no. 5, pp. 1340-1345, Oct. 2006.

[12] C. C. Collins, "Miniature passive pressure transensor for implanting in the eye," IEEE Trans. Biomed. Eng., vol. BME-14, no. 2, pp. 74-83, Apr. 1967.

[13] L. Rosengren, P. Rangsten, Y. Bäcklund, B. Hök, B. Svedbergh, and G. Selén, "A system for passive implantable pressure sensors," Sens. Actuators A, Phys., vol. 43, no. 1/3, pp. 55-58, May 1994.

[14] O. Akar, T. Akin, and K. Najafi, "A wireless batch sealed absolute capacitive pressure sensor," Sens. Actuators A, Phys., vol. 95, no. 1, pp. 29-38, Dec. 2001.

[15] A. DeHennis and K. D. Wise, "A double-sided single-chip wireless pressure sensor," in Proc. 15th IEEE Int. Conf. MEMS, Las Vegas, NV, Jan. 20-24, 2002, pp. 252-255.

[16] A. Baldi, W. Choi, and B. Ziaie, "A self-resonant frequency-modulated micromachined passive pressure transensor," IEEE Sensors J., vol. 3, no. 6, pp. 728-733, Dec. 2003.

[17] M. A. Fonseca, M. G. Allen, J. Kroh, and J. White, "Flexible wireless passive pressure sensors for biomedical applications," in Proc. 12th Solid-State Sens., Actuators, Microsyst. Workshop, Hilton Head Island, SC, Jun. 4-8, 2006, pp. 37-42.

[18] J. Shih, J. Xie, and Y.-C. Tai, "Surface micromachined and integrated capacitive sensors for microfluidic applications," in Proc. 12th Transducers Conf., Boston, MA, Jun. 8-12, 2003, pp. 388-391.

[19] P.-J. Chen, D. C. Rodger, S. Saati, M. S. Humayun, and Y.-C. Tai, "Implantable parylene-based wireless intraocular pressure sensor," in Proc. 21st IEEE Int. Conf. MEMS, Tucson, AZ, Jan. 13-17, 2008, pp. 58-61.

[20] M. A. Fonseca, J. M. English, M. von Arx, and M. G. Allen, "Wireless micromachined ceramic pressure sensor for high-temperature applications," J. Microelectromech. Syst., vol. 11, no. 4, pp. 337-343, Aug. 2002.

[21] F. E. Terman, Radio Engineers' Handbook. New York: McGraw-Hill, 1943.

[22] T. H. Lee, The Design of CMOS Radio-Frequency Integrated Circuits, 2nd ed. New York: Cambridge Univ. Press, 2004.

[23] S. Timoshenko and S. Woinowsky-Krieger, Theory of Plates and Shells, 2nd ed. New York: McGraw-Hill, 1959.

[24] A. P. Boresi and R. J. Schmidt, Advanced Mechanics of Materials, 6th ed. New York: Wiley, 2003.

[25] O. Tabata, K. Kawahata, S. Sugiyama, and I. Igarashi, "Mechanical property measurements of thin films using load-deflection of composite rectangular membranes," Sens. Actuators A, Phys., vol. 20, pp. 135-141, 1989.

[26] W. Sim, B. Kim, B. Choi, and J.-O. Park, "Theoretical and experimental studies on the parylene diaphragms for microdevices," Microsyst. Technol., vol. 11, no. 1, pp. 11-15, 2005.

[27] Certificate of Compliance, USP Biological Tests, North American Sci. Assoc., Inc., Northwood, OH, 2005.

[28] L. Wolgemuth, "Assessing the performance and suitability of parylene coating," Med. Device Diagn. Ind., vol. 22, pp. 42-49, 2000.

[29] X. Q. Wang, Q. Lin, and Y.-C. Tai, "A parylene micro check valve," in Proc. 12th IEEE Int. Conf. MEMS, Orlando, FL, Jan. 17-21, 1999, pp. 177-182.

[30] G. T. A. Kovacs, Micromachined Transducers Sourcebook. Boston, MA: McGraw-Hill, 1998.

[31] K. G. Ong, C. A. Grimes, C. L. Robbins, and R. S. Singh, "Design and application of a wireless, passive, resonant-circuit environmental monitoring sensor," Sens. Actuators A, Phys., vol. 93, no. 1, pp. 33-43, Aug. 2001

[32] J. Coosemans, M. Catrysse, and R. Puers, "A readout circuit for an intraocular pressure sensor," Sens. Actuators A, Phys., vol. 110, no. 1-3, pp. 432-438, Feb. 2004. 
[33] S. F. Pichorim and P. J. Abatti, "A novel method to read remotely resonant passive sensors in biotelemetric systems," IEEE Sensors J., vol. 8, no. 1, pp. 6-11, Jan. 2008.

[34] C. P. Yue and S. S. Wong, "On-chip spiral inductors with patterned ground shields for Si-based RF ICs," IEEE J. Solid-State Circuits, vol. 33, no. 5, pp. 743-752, May 1998.

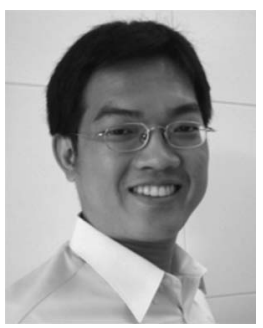

Po-Jui Chen (S'03) was born in Taipei, Taiwan, in 1980 . He received the B.S. degree in mechanical engineering from National Taiwan University, Taipei, in 2002, and the M.S. degree in electrical engineering from the California Institute of Technology (Caltech), Pasadena, in 2004, where he is currently working toward the Ph.D. degree in electrical engineering in the area of microelectromechanical systems (MEMS) in the Department of Electrical Engineering, Division of Engineering and Applied Science.

He has been with Dr. Yu-Chong Tai's Caltech Micromachining Laboratory as a Graduate Researcher since 2003. He has published more than 20 peerreviewed journal and conference papers and is the holder of several U.S. patent applications. His research interests include implantable biomedical microdevices, integrated microfluidic and laboratory-on-a-chip systems, MEMS sensors/actuators/structures, and micro-/nanofabrication technologies.

Mr. Chen is a student member of the American Society of Mechanical Engineers and a full member of Sigma Xi. He was elected as an honorary member of Phi Tau Phi in Taiwan in 2002. He is the recipient of several scholarships, the Sorenson Fellowship, the Outstanding College Youth Award at National Taiwan University in 2002, and the CHEMINAS Best Student Poster Award in the International MicroTAS 2006 Conference in Tokyo, Japan.

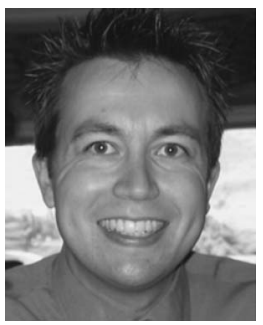

Damien C. Rodger (S'97-M'08) received the B.S. degree in electrical engineering (magna cum laude with honors) from Cornell University, Ithaca, NY, in 2000, and the Ph.D. degree in bioengineering from the California Institute of Technology, Pasadena, in 2008. He is currently working toward the M.D. degree in the Keck School of Medicine, University of Southern California, Los Angeles, while conducting research on microelectrode technologies for retinal and spinal cord prostheses and on other novel bioMEMS for ophthalmic use.

Dr. Rodger held a Whitaker Foundation Graduate Fellowship from 2003 to 2006 and is a member of the Association for Research in Vision and Ophthalmology.

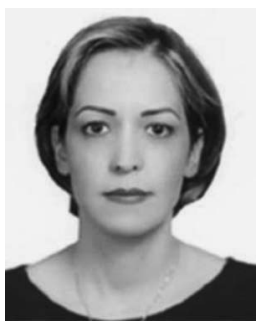

Saloomeh Saati received the M.D. degree from Iran University of Medical Sciences, Tehran, Iran, in 1999, and finished her training by completing an ophthalmology residency at Tehran University of Medical Sciences, Tehran, in 2005.

Currently, she is a Research Fellow of Ophthalmology with the University of Southern California, Los Angeles. She is also with the Doheny Eye Institute, Los Angeles, CA. She currently works on the Artificial Retina Project and intraocular bioMEMS implants, such as drug delivery devices, intraocular pressure sensors, and shunt drainage devices.

Ms. Saati is a member of the Association for Research in Vision and Ophthalmology.

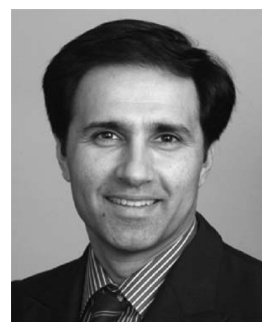

Mark S. Humayun (M'97) received the B.S. degree from Georgetown University, Washington, DC in 1984, the M.D. degree from Duke University, Durham, NC, in 1989, and the Ph.D. degree from the University of North Carolina, Chapel Hill, in 1994. He finished his training by completing an Ophthalmology Residency at Duke University and a Fellowship in Vitreoretinal Diseases at Johns Hopkins Hospital.

Currently, he is a Professor of Ophthalmology, Biomedical Engineering, and Cell and Neurobiology with the Keck School of Medicine, University of Southern California Los Angeles, where he is also the Director of the National Science Foundation Biomimetic Microelectronic Systems Engineering Research Center. He is also with the Doheny Eye Institute, Los Angeles. He is also the Director of the Department of Energy Artificial Retina Project that is a consortium of five Department of Energy laboratories and four universities, as well as industry.

Dr. Humayun is a member of the Association for Research in Vision and Ophthalmology, Biomedical Engineering Society, Vitreous Society, and Retina Society, and was elected as a Fellow of the American Institute for Medical and Biological Engineering in 2007.

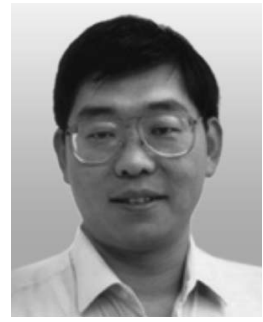

Yu-Chong Tai (M'97-SM'03-F'06) received the B.S. degree in electrical engineering from National Taiwan University, Taipei, Taiwan, in 1981, and the M.S. and Ph.D. degrees in electrical engineering from the University of California, Berkeley, in 1986 and 1989 , respectively.

$\mathrm{He}$ is with the Department of Electrical Engineering, Division of Engineering and Applied Science, California Institute of Technology (Caltech), Pasadena, where he founded the Caltech Micromachining Laboratory and currently is a Professor of Electrical Engineering, Mechanical Engineering, and Bioengineering. He is also with the Department of Bioengineering, Division of Engineering and Applied Science, Caltech. He has published more than 300 technical articles in the microelectromechanical systems (MEMS) field. His current research interests include flexible MEMS, bioMEMS, MEMS for retinal implants, parylenebased integrated microfluidics, neuroprobes/neurochips, and high performance liquid chromatography-based laboratory-on-a-chip systems.

Dr. Tai is the recipient of an IBM Fellowship, the David and Lucile Packard Fellowship, Ross Tucker Award, David Sakrison (Best Thesis Award), Presidential Young Investigator Award, Packard Award, Association for Laboratory Automation Achievement Award, Caltech Teaching Award, and several Prize Paper Awards. He cochaired the IEEE International MEMS 2002 Conference in Las Vegas, NV. He is a Fellow of the Institute of Physics. 